\title{
Ferromagnetism in nitrogen-doped MgO: Density-functional calculations
}

\author{
Phivos Mavropoulos, ${ }^{*}$ Marjana Ležaić, ${ }^{\dagger}$ and Stefan Blügel \\ Institut für Festkörperforschung (IFF), Institute for Advanced Simulation (IAS), and JARA-FIT, \\ Forschungszentrum Jülich, D-52425 Jülich, Germany
}

(Received 7 August 2009; revised manuscript received 5 October 2009; published 2 November 2009)

\begin{abstract}
The magnetic state of nitrogen-doped $\mathrm{MgO}$, with $\mathrm{N}$ substituting $\mathrm{O}$ at concentrations between $1 \%$ and the concentrated limit, is calculated with density-functional methods. The $\mathrm{N}$ atoms are found to be spin polarized with a moment of $1 \mu_{\mathrm{B}}$ per nitrogen atom and to interact ferromagnetically via the double-exchange mechanism in the full concentration range. The long-range magnetic order is established above a finite concentration of about $1.5 \%$ when the percolation threshold is reached. The disorder is described within the coherent-potential approximation, with the exchange interactions harvested by the method of infinitesimal rotations. The Curie temperature $T_{\mathrm{C}}$, calculated within the random-phase approximation, increases linearly with the concentration, and is found to be about $30 \mathrm{~K}$ for $10 \%$ concentration. Besides the substitution of single nitrogen atoms, also interstitial nitrogen atoms, dimers and trimers, and their structural relaxations are discussed with respect to the magnetic state. Possible scenarios of engineering a higher Curie temperature are analyzed, with the conclusion that an increase in $T_{\mathrm{C}}$ is difficult to achieve, requiring a particular attention to the choice of chemistry.
\end{abstract}

DOI: $10.1103 /$ PhysRevB.80.184403

PACS number(s): 75.50.Pp, 75.30.Hx, 75.30.Et

\section{INTRODUCTION}

In the research field of diluted magnetic semiconductors (DMS), a new direction is being investigated in the last five years, namely the engineering of ferromagnetic-state formation by $s p$ impurity doping. Compared to the more traditional DMS with transition-metal impurities, the novel $s p$ magnetism, or $d^{0}$ magnetism, is rather unexplored. The increasing interest is due to the perhaps unexpected finding that $p$ bands can spontaneously polarize giving a ferromagnetic state (although the possibility of magnetic $s p$ defects is long known) but also due to the hope of tuning the properties of these states so that high Curie temperatures are achieved even at low concentrations.

Theoretical considerations have revealed mainly two routes for the formation of $s p$-ferromagnetic states. In the first, the semiconductor or insulator cation is substituted by an atom of smaller valency, thus depriving the $p$-type valence band from electrons. This hole doping can shift the Fermi level into the valence band deep enough that the Stoner criterion is fulfilled and spontaneous spin polarization appears [see Fig. 1(a)]. This is, for example, the mechanism encountered in alkali-atom-doped $\mathrm{TiO}_{2}$ and $\mathrm{ZrO}_{2}$, predicted to be ferromagnetic by $a b$ initio calculations. ${ }^{1}$ Hole doping can also be achieved by cation vacancies, instead of substitution, as was, e.g., proposed for the cases of $\mathrm{HfO}_{2}, \mathrm{CaO}$, $\mathrm{ZrO}_{2}$, and $\mathrm{ZnO}{ }^{2-5}$

The second scenario is that the anion is substituted by one of smaller valency, introducing shallow, spin-polarized gap states. As the impurity concentration increases, these states form impurity bands, which remain spin polarized if the Stoner criterion is fulfilled [see Fig. 1(b)]. The magnetic moment is expected to be strongest for $2 p$ impurities, i.e., for the case in carbon- or nitrogen-doped oxides. ${ }^{5-14}$ This is because the $2 p$ states have no nodes and are rather localized, leading to a significant Hund's-type exchange interaction on site. In the concentrated limit (full substitution of the anion, see, e.g., Ref. 15), both routes converge to the same mechanism.
While the appearance of $p$-type magnetic states in oxides has been studied in the past, preliminary predictions of the Curie temperature have been so far based only on mean-field theory, ${ }^{6,7,12}$ which has since been shown to give qualitatively wrong results, overestimating $T_{\mathrm{C}}$ because it ignores the fundamental phenomenon of magnetic percolation in diluted magnetic systems. ${ }^{16-18}$

The scope of this paper is to investigate the appearance and stability of the ferromagnetic state, including calculations for the Curie temperature, in $\mathrm{MgO}_{1-x} \mathrm{~N}_{x}$ compounds for $x<15 \%$, in view of recent experimental activities with respect to the particular system. ${ }^{19}$ Our focus is on the solution provided by local density-functional theory (DFT), presented in Sec. II, and on the calculation of the Curie temperature (Sec. III) with the exchange constants harvested within the

(a) Cation substitution

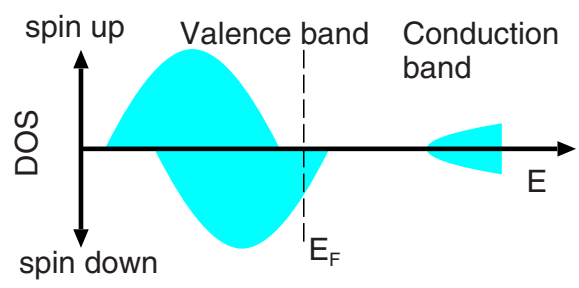

(b) Anion substitution

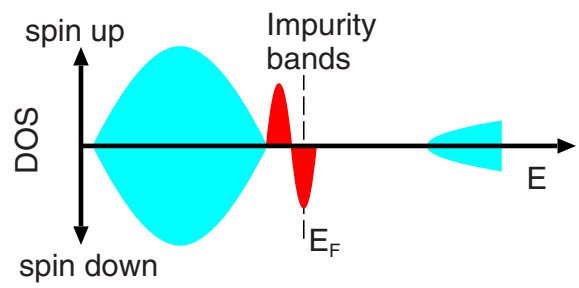

FIG. 1. (Color online) Typical mechanisms for $s p$ ferromagnetism: (a) cation substitution drives the Fermi level $E_{\mathrm{F}}$ into the valence band; (b) anion substitution creates an impurity band in the gap. 


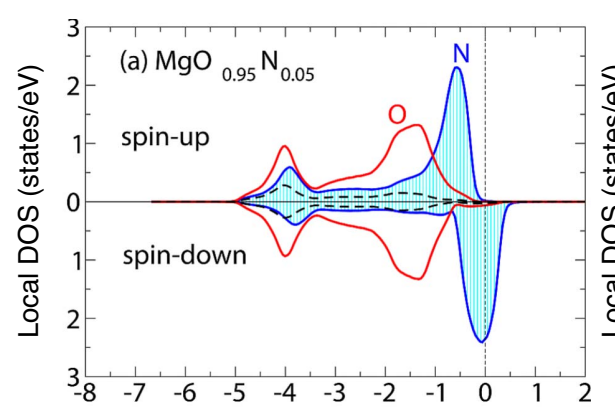

(a)

Energy relative to $E_{F}[e V]$

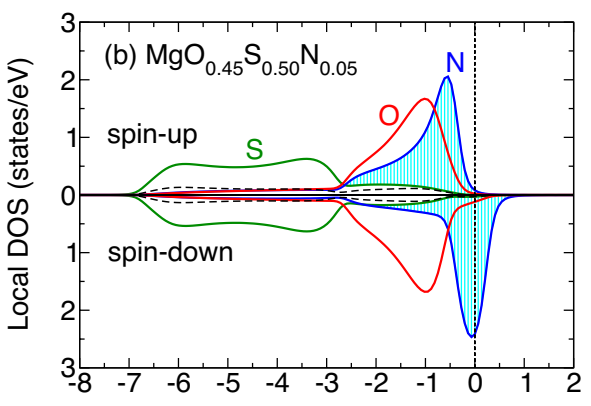

(b) Energy relative to $\mathrm{E}_{\mathrm{F}}[\mathrm{eV}]$

FIG. 2. (Color online) (a) Atom-resolved LDOS of $\mathrm{MgO}_{0.95} \mathrm{~N}_{0.05}$ calculated within the KKR-CPA. The LDOS of the Mg atom is presented by a dashed line. (b) Same for $\mathrm{MgO}_{0.45} \mathrm{~S}_{0.5} \mathrm{~N}_{0.05}$.

adiabatic approximation. As it turns out, for any reasonable concentration the Curie temperatures are significantly lower than room temperature, therefore, we also explore the possibility of increasing the Curie temperature by heavy doping in Sec. IV. Further on, we discuss the physics that lies beyond our approximations in Sec. V. There, among our considerations, a comparison is made to previously calculated results for $\mathrm{N}$-doped $\mathrm{MgO}$ that focus on the effects of electron correlation, and we also explore the constraints imposed by our specific structural model, including a discussion on the solubility of $\mathrm{N}$ in $\mathrm{MgO}$. Finally, we give an outlook in Sec. VI. The methods of calculation are shortly described in the Appendix.

\section{GROUND-STATE ELECTRONIC AND MAGNETIC STRUCTURES}

$\mathrm{MgO}$ crystallizes in the rock-salt structure with a lattice parameter of $4.21 \AA$ and exhibits a wide band gap of $7.8 \mathrm{eV}$. In our density-functional calculations the gap is found to be $4.8 \mathrm{eV}$, due to the well-known underestimation of insulator band gaps within local density-functional theory [localdensity approximation (LDA) or generalized gradient approximation (GGA)]. When substituting $\mathrm{O}, \mathrm{N}$ induces $p$ states in the $\mathrm{MgO}$ gap, approximately $0.5 \mathrm{eV}$ above the valence-band edge. As $\mathrm{N}$ has one electron less than $\mathrm{O}$, the gap states host one unpaired hole, showing a magnetic moment of $1 \mu_{\mathrm{B}}$ per $\mathrm{N}$ atom.

At finite concentrations, interaction among the impurity states forms a partially filled impurity band. The spin polarization remains present even at high concentrations, showing that magnetism is not only a consequence of the missing electron but also of the relatively strong Hund's exchange due to the localization of the $2 p$ states. The spin-polarized density of states shows a half-metallic behavior, as we found by calculations with the Korringa-Kohn-Rostoker (KKR) Green's-function method ${ }^{20}$ within the coherent-potential approximation (CPA) to disorder. (Details on the calculation methods are given in the Appendix.) Through a calculation of structural relaxation around a $\mathrm{N}$ impurity, performed with the Vienna $a b$ initio simulation package (VASP) (Ref. 21) at $5 \%$ concentration in supercell geometry, we could rule out serious Jahn-Teller distortions around the impurity; the first neighbors relax outward by less that $2 \%$, including only a weak symmetry breaking along one of the three crystallographic axes. This leads to a small lifting of degeneracy of the impurity state, insignificant compared to the impurity bandwidth. The small atomic displacements also justify the use of CPA.

We turn now to the discussion of the electronic and magnetic structures. Figure 2(a) shows the atom-resolved local density of states (LDOS) at a $\mathrm{N}$ concentration of $x=5 \%$. Evidently $\mathrm{N}$ has a spin-split DOS and exhibits a local magnetic moment. The $\mathrm{N}$ majority-spin (spin-up) impurity band is fully occupied and the minority-spin (spin-down) impurity band is occupied by $2 / 3$. As a consequence, the total moment is $1 \mu_{\mathrm{B}}$ per $\mathrm{N}$ atom. At lower concentrations the DOS is very similar but with smaller impurity bandwidth $w$ ( $w$ depends on the concentration as $w \sim \sqrt{x}$ since $x$ represents an average number of impurity neighbors, and it is known from the tight-binding approximation that $w$ increases as the square root of the number of neighbors). The exchange splitting is on the order of $0.5 \mathrm{eV}$, giving an exchange integral of $I$ $=0.5 \mathrm{eV} / \mu_{\mathrm{B}}$, which is approximately half the one in $3 d$ transition metals.

Already from examining the ferromagnetic density of states it is expected that the ferromagnetic state will be stable since $E_{\mathrm{F}}$ lies within the impurity band, favoring double exchange $^{22,23}$ (i.e., upon ferromagnetic alignment, hybridization leads to a broadening of the partially filled impurity band, resulting in energy gain). We confirmed this by calculations (not shown here in detail) of the total energy of the ferromagnetic state, $E_{\text {ferro, }}$, versus the disordered-localmoment state energy, $E_{\mathrm{DLM}}$; the latter is represented in the CPA by an alloy of the type $\mathrm{MgO}_{1-x} \mathrm{~N}_{0.5 x}^{\uparrow} \mathrm{N}_{0.5 x}^{\downarrow}$, where $\mathrm{N}^{\uparrow}$ and $\mathrm{N}^{\downarrow}$ are impurities with magnetic moment pointing "up" and "down." 22 As is typical for double-exchange DMS systems, ${ }^{16}$ we found that the energy gain of the ferromagnetic state, $E_{\text {DLM }}-E_{\text {ferro }}$, scales with the square root of the concentration. Further confirmation on the ferromagnetic nature of the ground state comes from calculations of the exchange constants, to be presented in the next section.

The spin-polarization energy (i.e., the energy gain of the system due to the moments' formation) drops with concentration due to the increase in hybridization, ranging between 550 and $100 \mathrm{meV}$ for $2 \%<x<15 \%$, as can be seen in Fig. 3 . However, a high spin-polarization energy is a necessary but not sufficient condition for the stability of the ferromagnetic 


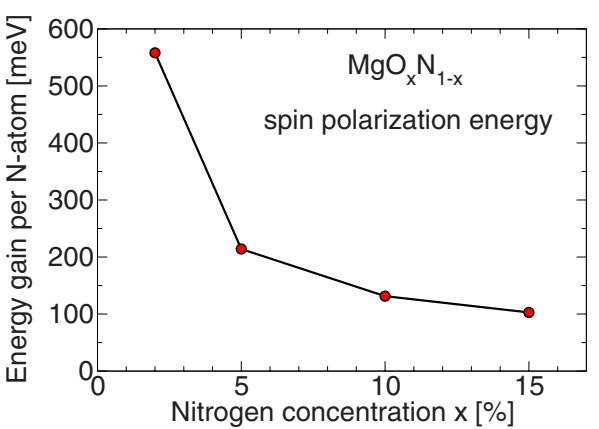

FIG. 3. (Color online) Spin-polarization energy per nitrogen atom as a function of concentration $x$. As the concentration increases, the hybridization increases, the impurity band becomes more itinerant, and the spin-polarization energy per atom drops. The line is a guide to the eyes.

state and for a high Curie temperature. The extension of the impurity gap state is also important, as it dictates the "communication" between localized moments and determines the onset of magnetic percolation. ${ }^{16,17}$ We therefore show in Fig. 4(a) the extension of the nitrogen-induced spin-polarized hole (calculations here were done within the KKR impurityin-host approach, assuming that the three $\mathrm{N}$-induced gap states are degenerate and occupied by $2 / 3$ each). We find that approximately half of the hole is localized at the impurity site while most of the remaining charge is distributed at the 12 nearest oxygen neighbors. From Fig. 4(b) it can also be seen that the hole falls off exponentially with distance, showing, however, a higher value in the $\langle 110\rangle$ directions. These are the directions of the first oxygen neighbors in the fcc lattice (which is the sublattice formed by oxygen atoms in $\mathrm{MgO}$ ), and in these directions also the pair exchange constants fall off slower (see Sec. III). The hole occupation at $\mathrm{Mg}$ sites is negligible.

\section{EXCHANGE INTERACTIONS AND CURIE TEMPERATURE}

We turn now to the discussion of the Curie temperature. We describe the fluctuations of the magnetization on the basis of the classical Heisenberg Hamiltonian,

$$
H=-\sum_{n n^{\prime}} J_{n n^{\prime}} \hat{e}_{n} \cdot \hat{e}_{n^{\prime}} .
$$

Here, $J_{n n^{\prime}}$ are the pair exchange parameters between $\mathrm{N}$ impurities at sites $n$ and $n^{\prime}$, and $\hat{e}_{n}$ and $\hat{e}_{n^{\prime}}$ are unit vectors pointing in the direction of the local moments. The Heisenberg model includes the transversal degrees of freedom of the fluctuating magnetic moments. The $J_{n n^{\prime}}$ are extracted from LDA results by the method of infinitesimal rotations ${ }^{24}$ (see the Appendix for details). As we see below, this energy scale (order of $10 \mathrm{meV}$ or less) is much smaller than the spin-polarization energy (order of $100 \mathrm{meV}$ or more), and thus the longitudinal fluctuations of the magnetic moments can be safely ignored as regards the Curie point.

The results are shown in Fig. 5. Clearly, all interactions are ferromagnetic. We note that there is a marked concentra-
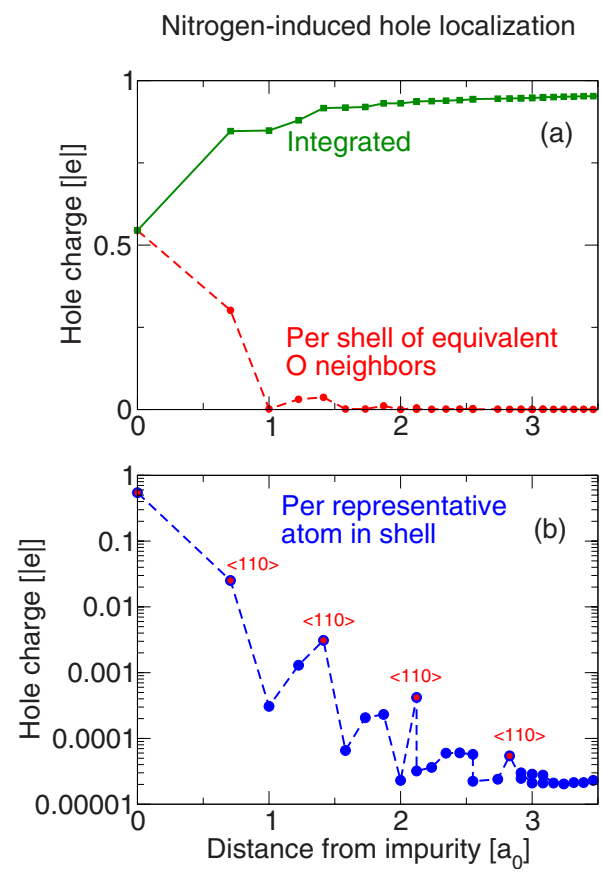

FIG. 4. (Color online) Spatial distribution of the nitrogeninduced spin-polarized hole for the case of a single nitrogen impurity substituting $\mathrm{O}$ in $\mathrm{MgO}$. Only the fraction of the hole at the $\mathrm{N}$ and $\mathrm{O}$ atoms is presented (the fraction at the $\mathrm{Mg}$ atoms is negligible). The distribution of the magnetic moment $\left(1 \mu_{\mathrm{B}}\right)$ is practically the same. (a) Full (green) line: Integrated hole charge as a function of distance from the impurity. Dashed (red) line: Hole charge per shell of $\mathrm{O}$ atoms that are equidistant from $\mathrm{N}$ and equivalent by the point-group symmetry. The impurity accommodates more than half of the hole charge. (b) Hole charge per atom as a function of distance from the impurity. Only one of the symmetry-equivalent atoms is considered for every shell. The points labeled $\langle 110\rangle$ represent atoms in the $\langle 110\rangle$ direction, in which the extent of the impurity wave function is largest. The lattice parameter is denoted by $a_{0}$. The lines are guides to the eyes.

tion dependence, with stronger exchange constants at low concentrations, as is well known in DMS systems with double-exchange ferromagnetic interactions (see, for example, Ref. 25).

It is interesting to analyze the exchange interactions, revealing that they are actually the net result of the ferromagnetic double exchange competing against a "hidden" antiferromagnetic superexchange. The energy-gaining mechanism of the latter is the following: if two impurities align antiferromagnetically, the majority-spin states of each impurity are shifted downward in energy as they hybridize with minorityspin states of the other. ${ }^{25}$ This superexchange becomes weaker if the impurity bands of at least one spin are far away from $E_{\mathrm{F}}$ (because then the hybridization is weaker). Hence the following trick was used to reveal the strength of the superexchange; we performed a nonself-consistent calculation of $J_{n n^{\prime}}$ (just one iteration) after driving the majority-spin $\mathrm{N}$ band away from the Fermi energy, by shifting the majority-spin potential of $\mathrm{N}$ to lower energies by $2.7 \mathrm{eV}$. The result (not shown here) was striking; these artificial $J_{n n^{\prime}}$, containing now only the double-exchange part, became stronger by approximately $50 \%$ even at long distances, showing that 

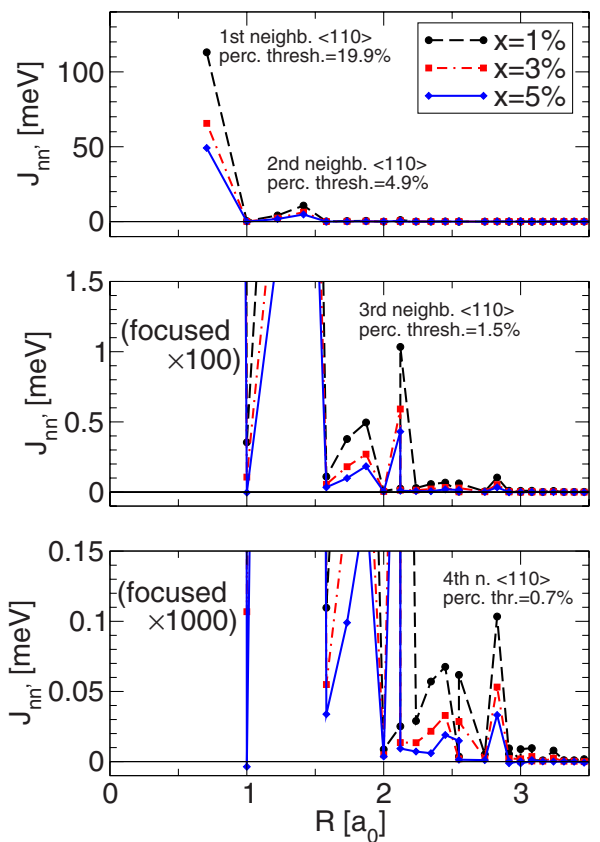

FIG. 5. (Color online) Pair exchange interactions $J_{n n^{\prime}}$ as a function of distance $R$ between nitrogen atoms for $x=1 \%, 3 \%$, and $5 \%$. The peaks corresponding to neighbors in the $\langle 110\rangle$ directions are indicated, together with the percolation threshold for interactions up to the particular distance. The middle and bottom panels show the same results at a different scale (focused 100 and 1000 times, respectively). The lattice parameter is denoted by $a_{0}$. The lines are guides to the eyes.

the superexchange part in the true $J_{n n^{\prime}}$ is significant.

It is evident that the nearest-neighbor exchange constants are large (Fig. 5), though insignificant, in the dilute case, for the stability of the ferromagnetic state; this is governed by longer-range exchange, due to the requirement for magnetic percolation. ${ }^{16,17}$ This means that, at low concentrations, the average interimpurity distance is large so that the short-range exchange interactions cannot produce a high Curie temperature although they are strong. Moreover, the $J_{n n^{\prime}}$ fall off exponentially with distance, as is expected for materials where the Fermi level falls in the gap at least for the one spin direction, ${ }^{26}$ resulting in a low $T_{\mathrm{C}}$ as we show below. As expected, the slowest decay of $J_{n n^{\prime}}$ is observed along $\langle 110\rangle$, i.e., in the directions where the charge of the impurity gap state falls off with the slowest rate (Fig. 4). Notably, the interactions in the $\langle 110\rangle$ directions are also dominating in zinc-blende or diamond structure DMS with transitionelement impurities. ${ }^{27,28}$

Because of the significance of magnetic percolation, we give in Fig. 5 also the percolation thresholds which we calculated for interaction distances corresponding to "peaks" of $J_{n n^{\prime}}$ in the $\langle 110\rangle$ directions. For example, we see that the nearest-neighbor coupling starts playing a role only at high concentrations, above $x=20 \%$ while the next peak, corresponding to $R \approx 1.41 a_{0}$, becomes relevant only for concentrations $x>4.9 \%$. Finally, we consider that interactions for distances higher than the third neighbor $\langle 110\rangle$ are negligibly small. This distance becomes relevant only for $x>1.5 \%$, thus we recognize $x=1.5 \%$ as the magnetic percolation threshold in $\mathrm{MgO}_{1-x} \mathrm{~N}_{x}$.

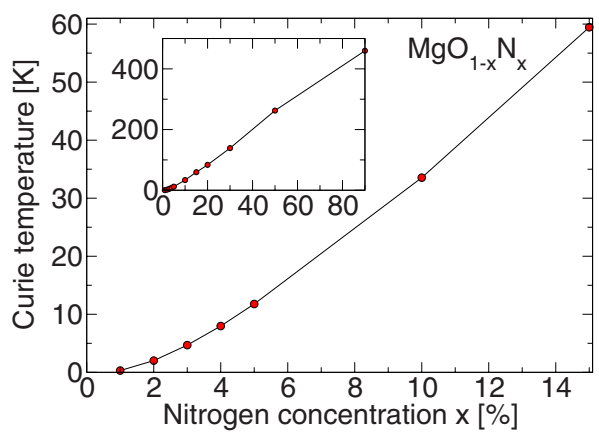

FIG. 6. (Color online) Curie temperatures for $\mathrm{N}$ concentrations between $1 \%$ and $15 \%$, calculated within the RPA. Inset: Same in the concentration range below $1-90 \%$. The lines are guides to the eyes.

A strong exponential decrease in $J_{n n^{\prime}}$ is an indication of a low Curie temperature in diluted magnetic systems. We have calculated $T_{\mathrm{C}}$ within the random-phase approximation (RPA) for diluted systems, following the prescription of Hilbert and Nolting ${ }^{18}$ (see the Appendix for calculational details). In doing this, we assumed the limit of classical spins $(S \rightarrow \infty$, with appropriate renormalization of the exchange constants), for reasons that we discuss in Sec. V. Although in this work we are interested in concentrations up to the order of $x$ $=10-15 \%$, in the calculation we included also high concentrations, in order to see the trend when approaching the concentrated limit. In special cases we also performed Monte Carlo calculations, as described in the Appendix, which gave typically $50 \%$ higher $T_{\mathrm{C}}$.

The results on the Curie temperature, as a function of concentration, are shown in Fig. 6. Starting from a percolation threshold at approximately $x=1.5 \%, T_{\mathrm{C}}$ rises linearly with concentration. Although, as we saw, the exchange constants at any particular distance weaken with increasing concentration, the important effect here comes from the decrease in the average interimpurity distance at higher $x$, allowing for the shorter-ranged stronger interactions to play a role in the ferromagnetic ordering.

At concentrations that are usually considered in DMS systems, i.e., not higher than $10-15 \%$, the Curie temperature turns out to be rather low; we find $T_{\mathrm{C}}=35 \mathrm{~K}$ at $10 \%$, and even at $x=20 \%, T_{\mathrm{C}}$ is still below $100 \mathrm{~K}$. In the following section we consider possible ways to achieve a higher Curie temperature.

\section{CONSIDERATIONS ON HOW TO INCREASE $T_{\mathrm{C}}$}

The low Curie temperature of $\mathrm{MgO}_{1-x} \mathrm{~N}_{x}$ is a clear drawback for application purposes at room temperature. In the interest of applications, one wonders if there could be some sort of material engineering to $\mathrm{MgO}_{1-x} \mathrm{~N}_{x}$ so that higher $T_{\mathrm{C}}$ can be achieved.

Since the Curie temperature of diluted magnetic systems depends on the long-range exchange constants, we have examined several possibilities of increasing $J_{n n^{\prime}}$ at distances larger than nearest neighbors. Considering that $J_{n n^{\prime}}$ falls off exponentially with distance $R_{n n^{\prime}}=\left|\vec{R}_{n}-\vec{R}_{n^{\prime}}\right|$ with a character- 
istic decay parameter $\kappa, J_{n n^{\prime}} \sim \exp \left(-\kappa R_{n n^{\prime}}\right)$, the main scope is to reduce $\kappa$. As we discuss here, we explored several routes to this end, tuning the band structure in ways that could be realistically achievable. However, none of these routes produced a significant improvement.

\section{A. Reducing the band gap}

Perhaps the most obvious way to reduce $\kappa$ is to engineer a smaller gap by alloying. For instance, $\mathrm{Mg}_{1-x} \mathrm{Zn}_{x} \mathrm{O}$ shows a smaller gap while retaining the rock-salt structure at not too high $\mathrm{Zn}$ concentrations; numerous other alloying combinations could have a similar effect. We pursued this idea by acting with an attractive constant potential on the $\mathrm{Mg}$ site, thus artificially reducing the $\mathrm{MgO}$ gap size. We then derived $\kappa(E)$ by calculating the complex band structure for energies in the gap region, i.e., looking for solutions of the Schrödinger equation that fall off as $\psi \sim e^{-\kappa r}$ (this procedure is well known in the calculation of surface and interface states). For $E$ deep in the gap there is a significant reduction in $\kappa(E)$ with the gap size. However, for $E$ at the actual position of the $\mathrm{N}$ levels (relatively close to the valence-band edge $\left.E_{v}\right), \kappa(E)$ is mainly determined by the effective mass $m^{*}$ of the light-hole valence band as $\kappa \approx \sqrt{2 m^{*}\left(E-E_{v}\right)} / \hbar$, due to the analytical behavior of the complex band structure. (Note that, among the complex bands, we seek the one with smallest value of $\kappa$, which corresponds to the band derived from the light holes. ${ }^{29}$ ) As the effective mass does not change appreciably, we find that a reduction in the gap does not affect $\kappa\left(E_{\mathrm{F}}\right)$ significantly, thus it cannot change the longrange exchange coupling.

\section{B. Changing the effective mass by compression}

After the previous considerations, the next obvious idea is to reduce the effective mass by compression, to be achieved, e.g., by epitaxial stress or strain. Calculations for a $5 \%$ reduction in the lattice constant show that only the effective mass of the heavy holes is appreciably affected while the light-hole bands are not much altered. However, what is important for a small $\kappa$ is the light-hole behavior; thus $\kappa\left(E_{\mathrm{F}}\right)$ does not change practically, and there is no increase in $J_{n n^{\prime}}$. Actually for large distances we do obtain small changes in $J_{n n^{\prime}}$ after compression but toward antiferromagnetic behavior. This has probably two origins: ${ }^{30}$ at smaller lattice constants, the bandwidth increases and the exchange splitting becomes smaller so that (i) the spin-down impurity band merges more with the valence-band setting on a RudermanKittel-Kasuya-Yosida (RKKY) behavior and (ii) the antiferromagnetic superexchange, which is sensitive to the exchange splitting, increases.

\section{Alloying with Sulfur}

Next we considered shifting the $\mathrm{N}$ impurity states closer to the valence band by proper alloying of $\mathrm{MgO}$ with a third component. In this respect, $\mathrm{MgO}_{1-y} \mathrm{~S}_{y}$ seemed promising because $\mathrm{MgS}$ also crystallizes in the rock-salt structure, with a larger lattice parameter $(5.2 \AA)$ and a smaller, indirect band gap (experimental: $4.5 \mathrm{eV}$; we find $2.25 \mathrm{eV}$ within the
LDA). Calculations in $\mathrm{MgO}_{1-y} \mathrm{~S}_{y}$ (which were carried out by changing the lattice constant according to Vegard's law) showed that the $\mathrm{O}$ valence states end up higher than the $\mathrm{S}$ valence states [see Fig. 2(b)]. This result is counterintuitive if one takes into account only the electronegativity of the elements. We interpret it by observing that the stronger localization of the $\mathrm{O}$ and $\mathrm{N} 2 p$ states is responsible for a stronger on-site Coulomb repulsion so that filled $2 p$ levels end up higher in energy than the more extended S $3 p$ levels. As a consequence, the valence-band edge in $\mathrm{MgO}_{1-y-x} \mathrm{~S}_{y} \mathrm{~N}_{x}$ is again oxygen dominated but the position of the $\mathrm{N}$ impurity band is closer to the oxygen valence-band edge. Thus there is a stronger hybridization of the $\mathrm{N}$ with the $\mathrm{O}$ states compared to $\mathrm{MgO}_{1-x} \mathrm{~N}_{x}$, as can be seen in Figs. 2(a) and 2(b). Because of this, the pair exchange constants are more extended in $\mathrm{MgO}_{1-y-x} \mathrm{~S}_{y} \mathrm{~N}_{x}$. Calculations of $\mathrm{MgO}_{0.45} \mathrm{~S}_{0.5} \mathrm{~N}_{0.05}$ showed an enhancement of $J(R)$ at large distances by up to 10-20\% compared to $\mathrm{MgO}_{0.95} \mathrm{~N}_{0.05}$. Thus the Curie temperature increases by a factor of the same order, remaining low.

\section{Doping with holes}

In principle, the double-exchange mechanism for ferromagnetism is most effective when the impurity band of one spin is exactly half filled while for the opposite spin is completely full (or empty). Then the energy gain in the ferromagnetic state by band broadening is maximized. In $\mathrm{MgO}_{1-x} \mathrm{~N}_{x}$ this is not the case, as the $\mathrm{N}$ spin-down band is filled by $2 / 3$. Therefore, doping with holes could help, as $E_{\mathrm{F}}$ will be shifted downward to the middle of the impurity band. Note that we are not trying to achieve the analog of Zener's holemediated $p$ - $d$ exchange but rather an improvement of the double-exchange mechanism. (Similar engineering has been proposed in transition-metal-doped DMS. ${ }^{31}$ ) We investigate this effect in a $\mathrm{Mg}$-poor compound, i.e., $\mathrm{Mg}_{1-y} \mathrm{O}_{1-x} \mathrm{~N}_{x}$. Each $\mathrm{Mg}$ vacancy adds two holes; thus the "ideal" Mg-vacancy concentration, resulting in exactly half filling of the $\mathrm{N}$ spindown band, is $y=x / 4$. In our calculations we used $x=10 \%$ and $y=2.5 \%$. As it turns out, the exchange interactions become more extended in space, however, the reason is that $E_{\mathrm{F}}$ now slightly enters the $\mathrm{O}$ valence band (since the valenceband top is anyhow merged with the lower part of the impurity band), and a Fermi surface is now available for the impurity interaction. As a result, a RKKY mechanism emerges, with an outcome of also antiferromagnetic interactions at large distances. The latter turn out to be comparatively strong, once more because of an additional increase in superexchange, as $E_{\mathrm{F}}$ shifts closer to the spin-up band. Thus there is no increase in $T_{\mathrm{C}}$. Tests with lower hole-doping concentration, before the RKKY exchange sets in, result in only a marginal increase in the long-range interactions, insufficient for a significant increase in $T_{\mathrm{C}}$.

\section{DISCUSSION ON APPROXIMATIONS}

\section{A. Approximations in the calculation of the electronic structure and Curie temperature}

We now consider which approximations used in our calculations could most seriously affect the electronic structure, 
exchange constants, and Curie temperature. Perhaps the most critical is the local-density approximation. Corrections that could qualitatively change the electronic structure include the self-interaction correction, inclusion of strong on-site Coulomb repulsion or $G W$ type of self-energy.

In Ref. 11, Droghetti et al. applied the self-interaction correction (SIC) to $\mathrm{MgO}$ doped with $\mathrm{N}$ (among other compounds). They find that an application of SIC to both $\mathrm{N}$ and $\mathrm{O}$ atoms increases the gap, as is expected by driving the occupied levels lower by the SIC. However, their main finding connected to our present discussion is the occurrence of a strong splitting on the order of $3 \mathrm{eV}$ between the occupied and unoccupied $p$ states of $\mathrm{N}$. The spin-down impurity band is, after SIC, manifestly insulating. A similar effect was found within the $\mathrm{LDA}+U$ method, applied by Pardo and Pickett $^{12}$ to a number of oxides (including $\mathrm{MgO}$ ) with $\mathrm{N}$ substitution of oxygen. In the latter work, a Coulomb repulsion of $U=5.5 \mathrm{eV}$ was used on the $\mathrm{O}$ and $\mathrm{N}$ atoms. From the point of view of ferromagnetism, an insulating impurity band can have severe consequences, in particular, disfavoring the double-exchange mechanism (impurity-band broadening does not lead to energy gain any more); ferromagnetic superexchange could be present but it is much weaker. In these cases, the system could even show a spin-glass ground state instead of a ferromagnetic one.

These works demonstrate possible consequences of strong Coulomb interactions, showing that local density-functional theory could be insufficient. We believe that, at low concentrations, these results undoubtedly show the correct physics but at higher concentrations (a few percent) they possibly overestimate the Coulomb interaction, and are therefore still inconclusive as to the exact nature of the ground state. Our arguments are as follows.

First, considering the low-concentration limit, the $\mathrm{N}$ impurity states are not localized at the $\mathrm{N}$ atomic cell but rather extended also over the 12 nearest oxygen neighbors, as our calculation shows. This speaks for a relatively mild selfinteraction error, compared to $d$ or $f$ systems, where the SIC is usually applied to. The LDA error must become less and less serious as the concentration increases and an itinerant impurity band is formed. Furthermore, application of Coulomb correction terms on the oxygen valence band is probably much less necessary, as the associated Bloch states are itinerant with a significant bandwidth (in the limiting case of completely nonlocalized states of a homogeneous electron gas, the self-interaction is fully corrected by the LDA exchange-correlation energy). We conclude that Coulombinteraction corrections should be of different scale at the $\mathrm{N}$ impurity-band states compared to the $\mathrm{O}$ valence-band states but even for $\mathrm{N}$ they should not be too severe.

Second, as the concentration $x$ increases, the impurity bandwidth $w \sim \sqrt{x}$ increases rather fast, exceeding $1 \mathrm{eV}$ already at $x=5 \%$. This bandwidth can well be on the order of magnitude of the local Coulomb interaction $U$ (in the LDA $+U$ calculations of Ref. 12, a value of $U=5.5 \mathrm{eV}$ was used, which, however, acts on the atomic site; if the full extent of the impurity wave function is accounted for, a significantly lower value of $U$ would be needed for the same effect). In this case a Mott transition is possible, from an insulating state for low concentrations (where $w<U$ ) to a metallic state at high concentrations (where $w>U$ ). In such a scenario, ferromagnetism would be assisted by the increase in concentration in two ways: occurrence of a metallic state similar to the LDA result and magnetic percolation.

Concerning $G W$ type of corrections, these are known to correct the LDA underestimation of band gap in band insulators. It should be then expected that the larger gap would lead to a stronger decay of the exchange constants with distance, reducing the calculated $T_{\mathrm{C}}$. However, this reduction should not be too serious, for the same reason that $T_{\mathrm{C}}$ cannot be much increased by engineering a smaller gap (see Sec. IV).

Another approximation that was made here was the assumption of a classical, rather than quantum, Heisenberg model to describe the magnetic excitations. Considering that we are faced with an $S=\frac{1}{2}$ system, it can be argued that a classical approximation is unrealistic. Within the RPA, a change from a classical to a quantum Heisenberg model leads to an increase in $T_{\mathrm{C}}$ by a factor of $S(S+1) / S^{2}=3$, in the $S=\frac{1}{2}$ case. Accepting this, room-temperature ferromagnetism could be achieved at $x=20 \%$, as can be seen by scaling up the results of Fig. 6. However, the particular way of calculation of the exchange coupling constants ${ }^{24} J_{n n^{\prime}}$ tacitly assumes a classical model. The constants are calculated within constrained density-functional theory, in principle, by "freezing" the system in a static noncollinear configuration and calculating the total energy; this should be viewed as a parametrization of the low-energy excitations of the spin density, rather than a derivation of a quantum spin Hamiltonian. Comparison to experiments in previous works also advocates for this point of view. E.g., Sasioglu et al. ${ }^{32}$ have calculated the Curie temperature of Heusler alloys by a similar recipe. While the assumption of a classical Heisenberg model lead them to reasonable agreement of $T_{\mathrm{C}}$ with experiment, the assumption of a quantum model resulted in a clear overestimation of $T_{\mathrm{C}}$. For lack of a better theory that predicts $J_{n n^{\prime}}$ for a quantum Heisenberg model, we are obliged to work within a classical model.

\section{B. Approximations imposed by our structural model}

So far we assumed a uniform, on the average, distribution of the $\mathrm{N}$ atoms in the $\mathrm{MgO}$ matrix. We therefore start this section by commenting on the solubility of $\mathrm{N}$ in $\mathrm{MgO}$. The solubility limit of substitutional $\mathrm{N}$ is expected to be small and clustering of the $\mathrm{N}$ impurities is expected to be favored. We verified this by calculating, within the KKR-CPA, the mixing energy as a function of the concentration, $E_{\text {mix }}(x)$ $=E\left[\mathrm{MgO}_{1-x} \mathrm{~N}_{x}\right]-(1-x) E[\mathrm{MgO}]-x E[\mathrm{MgN}]$ with $\mathrm{MgN}$ in the rock-salt structure. $E_{\text {mix }}(x)$ was positive for all calculated concentrations $(1 \% \leq x \leq 90 \%)$, showing that phase separation into $\mathrm{MgO}$ and $\mathrm{MgN}$ is energetically favored. This can be understood also from the local density of states, shown in Fig. 2(a), where it is seen that the impurity band is bisected by the Fermi level $E_{\mathrm{F}}$. In case of impurity clustering the bandwidth will increase and energy will be gained because of the lowering of the occupied levels. Interestingly, the origin of ferromagnetic double exchange (bisection of the impurity band by $E_{\mathrm{F}}$ ) provides also a mechanism for (usually 
unwanted) phase separation. Of course, phase separation other than the $\mathrm{MgO}-\mathrm{MgN}$ type could be also possible; what this calculation shows is the thermodynamic instability of $\mathrm{MgO}_{1-x} \mathrm{~N}_{x}$ with respect to at least one type of phase separation, even if entropic effects could milden the separation. The preference toward clustering was also seen in calculations of two substitutional $\mathrm{N}$ impurities in a $\mathrm{MgO}$ supercell matrix; the most stable state was found when the two $\mathrm{N}$ atoms were first neighbors in the $\langle 110\rangle$ direction. We conclude that $\mathrm{MgO}_{1-x} \mathrm{~N}_{x}$ can only be grown under out-of-equilibrium conditions, as is the case with many transition-metal-doped DMS.

Two neighboring substitutional $\mathrm{N}$ atoms still provide a metallic state. However, three neighboring $\mathrm{N}$ atoms forming an equilateral triangle in a $\{111\}$ plane cause a transition to a semiconducting state (with a split minority-spin band), if structural optimization of the three atoms and their surroundings is taken into account.

Coming now to interstitial $\mathrm{N}$ impurities, our calculations (performed with VASP) showed that strong atom displacements are involved, thus we cannot use CPA to describe the random alloy with interstitials. As the phase space involved is extremely rich, and probably depends strongly on growth and annealing conditions, we defer a deeper analysis of the particular question to a future work, commenting on a few interesting findings.

First, in the case of a single $\mathrm{N}$ interstitial, the symmetric, tetrahedral position constitutes only a local minimum of the total energy. Further significant reduction in the total energy is found in a "dumbbell" configuration reminiscent of a NO molecule, where the $\mathrm{N}$ impurity binds itself to an $\mathrm{O}$ atom, with both atoms in the proximity of the ideal $\mathrm{O}$ lattice position. This entity is found to be half metallic and magnetic, with a moment of $1 \mu_{\mathrm{B}}$.

Second, when two nitrogen atoms are placed around an oxygen atom as interstitials, structural relaxation leads to a "zigzag" O-N-O-N configuration. Here, the two $\mathrm{N}$ atoms are almost on the lattice sites, being first neighbors along $\langle 110\rangle$ while the $\mathrm{O}$ atoms are shifted out of their ideal positions, hovering above the (110) atomic layer. The electronic structure changes to nonmagnetic and insulating.

Third, the following configuration is of particular interest: two neighboring $\mathrm{N}$ atoms together with an $\mathrm{O}$ interstitial between them, plus a nearby $\mathrm{O}$ vacancy. This is so to say a configuration where an $\mathrm{O}$ atom has been bound during growth by a $\mathrm{N}$ pair, missing the lattice position nearby. This configuration shows a local energy minimum and is nonmagnetic. However, if the $\mathrm{O}$ atom returns to the vacant lattice position, the state becomes magnetic with a total-energy gain on the order of $4 \mathrm{eV}$ while the lattice parameter is reduced by $1 \%$ (in the particular calculation we took a $\mathrm{Mg}_{32} \mathrm{O}_{30} \mathrm{~N}_{2}$ supercell, corresponding to $6.25 \% \mathrm{~N}$ ). This is a possible reason that magnetism appears after annealing in experiment. ${ }^{19}$

\section{CONCLUSIONS AND OUTLOOK}

The chemical compound $\mathrm{MgO}_{1-x} \mathrm{~N}_{x}$ in which oxygen is substituted by nitrogen atoms is found to be ferromagnetic for all concentrations which seem realistically achievable $(x \leq 15 \%)$ and above the concentration of the percolation threshold (about $1.5 \%$ ). The $\mathrm{N}$ atom forms a partially occupied gap state at the vicinity of the valence-band edge. This leads to concentration-dependent exchange parameters, exponentially decreasing with distance, which are ferromagnetic due to double exchange as dominating mechanism. The reduction in the average $\mathrm{N}-\mathrm{N}$ distance and the reduction in the exchange interaction strength with increasing concentration compensate partially so that the Curie temperature increases linearly with concentration. The structural relaxations around the $\mathrm{N}$ impurity are found to be small. In the case of $\mathrm{N}$ clusters and interstitial $\mathrm{N}$ we found magnetic as well as nonmagnetic complexes but never with higher moments than $1 \mu_{\mathrm{B}}$ /atom.

We think that the Curie temperature of such sp compounds can be increased if the exchange interactions become more long ranged, e.g., by moving the gap states closer to the band edge, and if the N-N exchange interactions are enhanced by suppressing the competing antiferromagnetic superexchange via an increase in the local exchange splitting. Our attempts in this direction, using compounds such as $\mathrm{MgO}_{1-x-y} \mathrm{~S}_{y} \mathrm{~N}_{x}$ or $\mathrm{Mg}_{1-y} \mathrm{O}_{1-x} \mathrm{~N}_{x}$, have not been met with success. Nevertheless, it is worthwhile to investigate more host/ impurity combinations theoretically, in order to guide experimental efforts.

From the point of view of applications, a low $T_{\mathrm{C}}$ is a limiting factor. However, from the point of view of physics, $s p$ magnetism in oxides presents intriguing open problems, such as the nature of the ground state, its concentration dependence, and the role of dynamic electron correlations. Moreover, although homogeneous nitrogen distributions at high concentrations are difficult to achieve, it is worthwhile to examine the physics and technological relevance of inhomogeneous samples, emerging, e.g., by spinodal decomposition ${ }^{33}$ or delta doping, ${ }^{28}$ as has been proposed for transition-metal-doped DMS. Such compounds could show much more robust magnetism locally, leading to functionalities.

\section{ACKNOWLEDGMENTS}

We would like to thank Stuart Parkin for discussions on his experimental results prior to publication. We are grateful to Peter H. Dederichs for his constant support and enlightening discussions and to Yuriy Mokrousov for a critical reading of the manuscript. This work was funded in part by the Young Investigators Group Programme of the Helmholtz Association ("Computational Nanoferronics Laboratory," Contract No. VH-NG-409).

\section{APPENDIX: METHODS OF CALCULATION}

For the calculations of the alloy ground-state electronic structure (except structural relaxations) we used the fullpotential KKR Green's-function method ${ }^{20}$ with exact treatment of the atomic cell shapes, ${ }^{34}$ using an angular momentum cutoff of $l_{\max }=3$ and a maximal $k$-point mesh of 64000 points in the full Brillouin zone; the complex-energy contour integration included the oxygen and nitrogen $2 s$ states. Dis- 
order was treated within the KKR-CPA. Relativistic effects were neglected. In the KKR-CPA calculations we used the $\mathrm{MgO}$ experimental lattice parameter for all concentrations, as the small change should have no significant effect in the calculated trends.

For the calculation of the hole localization, the impurityin-host version of the KKR method was employed nonselfconsistently, with potentials taken from self-consistent CPA. In the KKR-CPA calculations, the LDA for the exchangecorrelation energy was used with the parametrization of Vosko et al. ${ }^{35}$ For the structural relaxations we used the ${ }^{21}$ VASP projector-augmented wave code within the GGA. ${ }^{36}$

The exchange constants $J_{n n^{\prime}}$ were calculated within the approximation of infinitesimal rotations. ${ }^{24}$ This method is based on a hypothesis of correspondence of the energy change $\Delta H$ in the Heisenberg Hamiltonian (1) with the total-energy change found within DFT, $\Delta E_{\mathrm{DFT}}$, upon rotating the magnetic-moment directions $\hat{e}_{n}$ and $\hat{e}_{n^{\prime}}$. In addition, the magnetic force theorem is used, which states that the energy change due to infinitesimal rotations in the moments direction can be calculated by the difference in the Kohn-Sham eigenvalues. One obtains the exchange constants by taking a second derivative, $J_{n n^{\prime}}=-\frac{1}{2} \partial^{2} H /\left(\partial \hat{e}_{n} \partial \hat{e}_{n^{\prime}}\right)$ $=-\frac{1}{2} \partial^{2} E_{\mathrm{DFT}} /\left(\partial \hat{e}_{n} \partial \hat{e}_{n^{\prime}}\right), \mathrm{as}^{24}$

$$
\begin{aligned}
J_{n n^{\prime}}= & \frac{1}{4 \pi} \int^{E_{\mathrm{F}}} \operatorname{Im} \operatorname{Tr}_{l m} G_{n n^{\prime}}^{\uparrow}(E)\left[t_{n^{\prime}}^{\uparrow}(E)-t_{n^{\prime}}^{\downarrow}(E)\right] \\
& \times G_{n^{\prime} n}^{\downarrow}(E)\left[t_{n}^{\downarrow}(E)-t_{n}^{\uparrow}(E)\right] d E,
\end{aligned}
$$

where $G_{n n^{\prime}}^{\uparrow, \downarrow}(E)$ is the intersite KKR structural Green's function for spin up $(\uparrow)$ or down $(\downarrow), t_{n}^{\uparrow, \downarrow}$ are spin-dependent scattering matrices, and $\operatorname{Tr}_{l m}$ indicates a trace in angular momentum indices $\left(G_{n n^{\prime}}\right.$ and $t_{n}$ are matrices in angular momentum space). As has been found by Sato et al. ${ }^{37}$ the method of infinitesimal rotations within the CPA can overestimate the values of $J_{n n^{\prime}}$ between the nearest neighbors at low concentrations (when they anyhow are irrelevant for $T_{\mathrm{C}}$ ) but is rather good for larger distances.
The Curie temperatures were calculated within the RPA for diluted magnetic systems, as proposed by Hilbert and Nolting, ${ }^{18}$ with the distance-dependent interactions $J_{n n^{\prime}}$ taken as described above. Within this method, ${ }^{18}$ first a large, periodically repeated supercell is considered, including a few thousands of randomly distributed magnetic impurities. Then the RPA is used to find the eigenvalues $\hbar \omega_{i}$ of the magnetic excitation spectrum of the Heisenberg Hamiltonian. However, contrary to the application of RPA to concentrated systems, in this approach the lowest eigenvalues are not accounted for when calculating the Curie temperature. The reasoning is that these correspond to excitations of almost isolated magnetic atoms or clusters which become irrelevant in the thermodynamic limit. A cutoff $E_{\text {cut }}$ is introduced and one retains only the eigenvalues for which $\hbar \omega_{i}>E_{\text {cut }}$; the Curie temperature then reads, for classical spin systems,

$$
k_{\mathrm{B}} T_{\mathrm{C}}=\frac{2}{3}\left(\frac{1}{N_{\hbar \omega_{i}>E_{\mathrm{cut}}}} \sum_{\hbar \omega_{i}}\right)^{-1}
$$

with $k_{\mathrm{B}}$ the Boltzmann constant. Thus $T_{\mathrm{C}}$ is at first cutoff dependent; nevertheless, a natural $E_{\text {cut }}$ emerges at the point where the curve $T_{\mathrm{C}}\left(E_{\text {cut }}\right)$ levels off and reaches a plateau after an initial abrupt increase. As the simulation supercell becomes larger and the thermodynamic limit is approached, the $T_{\mathrm{C}}$ plateau is reached at ever lower $E_{\text {cut }}$.

Returning to the present calculations, at each concentration an environmental average was evaluated by taking 100 random configurations of approximately 8500 nitrogen atoms each, statistically distributed in a simulation supercell (sized between $21^{3}$ unit cells for $90 \%$ concentration and $94^{3}$ unit cells for $1 \%$ concentration). For each configuration the RPA yielded a $T_{\mathrm{C}}$, and an average of all values of $T_{\mathrm{C}}$ was determined at the end. In special cases $(x=5 \%, 10 \%, 15 \%$, and $20 \%$ ) the results were cross-checked with Monte Carlo calculations, which yielded a $T_{\mathrm{C}}$ of approximately $50 \%$ higher, except for $x=5 \%$ where the results of the two methods agreed. Because the Monte Carlo method is numerically more expensive, in these tests we treated systems of 10002500 magnetic ions, using up to 20 configurations for averaging; within Monte Carlo, $T_{\mathrm{C}}$ was calculated using the fourth-order cumulant method. ${ }^{38}$

\footnotetext{
*ph.mavropoulos@fz-juelich.de

†m.lezaic@fz-juelich.de

${ }^{1}$ F. Máca, J. Kudrnovský, V. Drchal, and G. Bouzerar, Appl. Phys. Lett. 92, 212503 (2008); Philos. Mag. 88, 2755 (2008).

${ }^{2}$ C. Das Pemmaraju and S. Sanvito, Phys. Rev. Lett. 94, 217205 (2005).

${ }^{3}$ J. Osorio-Guillén, S. Lany, S. V. Barabash, and A. Zunger, Phys. Rev. Lett. 96, 107203 (2006); Phys. Rev. B 75, 184421 (2007).

${ }^{4}$ G. Bouzerar and T. Ziman, Phys. Rev. Lett. 96, 207602 (2006).

${ }^{5}$ H. Peng, H. J. Xiang, S.-H. Wei, S.-S. Li, J.-B. Xia, and J. Li, Phys. Rev. Lett. 102, 017201 (2009).

${ }^{6}$ K. Kenmochi, M. Seike, K. Sato, A. Yanase, and H. KatayamaYoshida, Jpn. J. Appl. Phys. 43, L934 (2004).

${ }^{7}$ K. Kenmochi, V. A. Dinh, K. Sato, A. Yanase, and H. KatayamaYoshida, J. Phys. Soc. Jpn. 73, 2952 (2004).
}

${ }^{8}$ Van An Dinh, K. Sato, and H. Katayama-Yoshida, Solid State Commun. 136, 1 (2005).

${ }^{9}$ I. S. Elfimov, A. Rusydi, S. I. Csiszar, Z. Hu, H. H. Hsieh, H.-J. Lin, C. T. Chen, R. Liang, and G. A. Sawatzky, Phys. Rev. Lett. 98, 137202 (2007).

${ }^{10}$ V. V. Bannikov, I. R. Shein, and L. Ivanovskii, Tech. Phys. Lett. 33, 541 (2007).

${ }^{11}$ A. Droghetti, C. D. Pemmaraju, and S. Sanvito, Phys. Rev. B 78, 140404(R) (2008).

${ }^{12}$ V. Pardo and W. E. Pickett, Phys. Rev. B 78, 134427 (2008).

${ }^{13}$ B. Gu, N. Bulut, T. Ziman, and S. Maekawa, Phys. Rev. B 79, 024407 (2009).

${ }^{14}$ A. Droghetti and S. Sanvito, Appl. Phys. Lett. 94, 252505 (2009).

${ }^{15}$ M. Sieberer, J. Redinger, S. Khmelevskyi, and P. Mohn, Phys. 
Rev. B 73, 024404 (2006).

${ }^{16}$ K. Sato, W. Schweika, P. H. Dederichs, and H. KatayamaYoshida, Phys. Rev. B 70, 201202(R) (2004).

${ }^{17}$ L. Bergqvist, O. Eriksson, J. Kudrnovsky, V. Drchal, P. Korzhavyi, and I. Turek, Phys. Rev. Lett. 93, 137202 (2004).

${ }^{18}$ S. Hilbert and W. Nolting, Phys. Rev. B 70, 165203 (2004).

${ }^{19}$ S. S. P. Parkin, private communication; See also C.-H. Yang, M. Samant, and S. Parkin, APS March Meeting Abstract, 2009 (unpublished), p. V22.00004; http://meetings.aps.org/Meeting/ MAR09/Event/98745

${ }^{20}$ The SPR-TB-KKR package, H. Ebert and R. Zeller, http:// olymp.cup.uni-muenchen.de/ak/ebert/SPR-TB-KKR

${ }^{21}$ G. Kresse and J. Hafner, Phys. Rev. B 47, 558 (1993); G. Kresse, Thesis, Technische Universität Wien, 1993; G. Kresse and J. Furthmüller, Comput. Mater. Sci. 6, 15 (1996); Phys. Rev. B 54, 11169 (1996); G. Kresse and D. Joubert, ibid. 59, 1758 (1999); P. E. Blöchl, ibid. 50, 17953 (1994).

${ }^{22}$ H. Akai, Phys. Rev. Lett. 81, 3002 (1998).

${ }^{23}$ L. M. Sandratskii and P. Bruno, Phys. Rev. B 73, 045203 (2006).

${ }^{24}$ A. I. Liechtenstein, M. I. Katsnelson, V. P. Antropov, and V. A. Gubanov, J. Magn. Magn. Mater. 67, 65 (1987).

${ }^{25}$ B. Belhadji, L. Bergqvist, R. Zeller, P. H. Dederichs, K. Sato, and H. Katayama-Yoshida, J. Phys.: Condens. Matter 19, 436227 (2007).

${ }^{26}$ M. Pajda, J. Kudrnovský, I. Turek, V. Drchal, and P. Bruno, Phys. Rev. B 64, 174402 (2001).

${ }^{27}$ L. M. Sandratskii and P. Bruno, Phys. Rev. B 67, 214402 (2003); J. Kudrnovský, V. Drchal, I. Turek, L. Bergqvist, O. Eriksson, G. Bouzerar, L. Sandratskii, and P. Bruno, J. Phys.:
Condens. Matter 16, S5571 (2004); S. Picozzi and M. Ležaić, New J. Phys. 10, 055017 (2008).

${ }^{28}$ S. Picozzi, M. Ležaić, and S. Blügel, Phys. Status Solidi A 203, 2738 (2006).

${ }^{29}$ P. H. Dederichs, P. Mavropoulos, O. Wunnicke, N. Papanikolaou, V. Bellini, R. Zeller, V. Drchal, and J. Kudrnovský, J. Magn. Magn. Mater. 240, 108 (2002); P. Mavropoulos, N. Papanikolaou, and P. H. Dederichs, Phys. Rev. Lett. 85, 1088 (2000).

${ }^{30}$ L. Bergqvist, B. Belhadji, S. Picozzi, and P. H. Dederichs, Phys. Rev. B 77, 014418 (2008).

${ }^{31}$ T. Fukushima, K. Sato, H. K. Yoshida, and P. H. Dederichs, Physica B (Amsterdam) 376-377, 786 (2006).

${ }^{32}$ E. Sasioglu, L. M. Sandratskii, P. Bruno, and I. Galanakis, Phys. Rev. B 72, 184415 (2005).

${ }^{33}$ K. Sato, T. Fukushima, and H. Katayama-Yoshida, J. Phys.: Condens. Matter 19, 365212 (2007).

${ }^{34}$ N. Stefanou and R. Zeller, J. Phys.: Condens. Matter 3, 7599 (1991).

${ }^{35}$ S. H. Vosko, L. Wilk, and M. Nusair, Can. J. Phys. 58, 1200 (1980).

${ }^{36}$ J. P. Perdew, K. Burke, and M. Ernzerhof, Phys. Rev. Lett. 77, 3865 (1996).

${ }^{37}$ K. Sato, P. H. Dederichs, and H. Katayama-Yoshida, Phys. Status Solidi C 3, 4143 (2006).

${ }^{38}$ D. P. Landau and K. Binder, A Guide to Monte Carlo Simulations in Statistical Physics (Cambridge University Press, Cambridge, 2000). 